Results: A total of 2670 DEGs and 371 TSPJ targets were obtained, including 52 overlapping genes. 41 genes had protein interactions that are used to build the PPI network. The results of the KEGG enrichment analysis included VEGF and HIF-1 signaling pathway. Seven negative correlation genes and 16 positive correlation genes were obtained by correlational analysis of DEGs in VEGF and HIF-1 signaling pathway. SRC proto-oncogene, nonreceptor tyrosine kinase (SRC), and the signal transducer and the activator of transcription 3 (STAT 3) had a higher value of degree in PPI and showed a significant correlation in the pathways; they were regarded as key targets. Compared with the CIA model group, TSPJ significantly decreased the Al and histology scores. Moreover, the expression of VEGF-A, HIF-1 $\alpha$, IL-1 $\beta$, and IL-17A in serum or spleens significantly reduced in a dose-dependent.

Conclusion: Present study show that SRC and STAT 3 may be the key targets of TSPJ acting on the VEGF and HIF-1 signaling pathways, thus inhibiting angiogenesis and improving RA.

Disclosure of Interests: None declared

DOI: 10.1136/annrheumdis-2021-eular.3496

\section{AB0067 RHEUMATOID ARTHRITIS IS DRIVEN NOT ONLY BY INFLAMMATION BUT ALSO BY FIBROGENESIS}

A. C. Bay-Jensen ${ }^{1}$, S. F. Madsen ${ }^{1,2}$, K. Gehring ${ }^{3}$, K. Musa ${ }^{3}$, M. Karsdal' ${ }^{1}{ }^{1}$ Nordic Bioscience, ImmunoScience, Herlev, Denmark; ${ }^{2}$ University of Copenhagen, Department of Biomedical Sciences, Copenhagen, Denmark; ${ }^{3}$ Nordic Bioscience, Clinical Laboratory, H, Denmark

Background: Rheumatoid arthritis (RA) is a chronic, autoimmune disease characterized by inflammation of the synovium, cartilage and bone leading to joint swelling, tenderness, and dysfunction. The destruction of the joint tissue involves degradation of the extracellular matrix (ECM). The ECM consist of collagens and other connective proteins ${ }^{1}$. Studies have shown that elevated levels of collagen metabolites, such as those of type I, II, III and VI, are highly elevated in RA, correlated to disease activity and modulated in response to, for example tocilizumab ${ }^{2}$, baricitinib $^{3}$ and methotrexate ${ }^{4}$. However, little is known about the formation of collagen, fibroblast activity, the fibrotic component of RA and how this influence likelihood of response to treatment.

Objectives: We investigated the level of active fibrogenesis in a population of moderate to severe RA patients (in contrast healthy controls) by assessing blood-levels of PRO-C3 and PRO-C6 (type III and VI collagen formation markers), which have been reported to be associated with the degree and extent of fibrosis5.

Methods: PRO-C3 and PRO-C6 was measured in serum of 166 RA patients (age; 54 (20-82), $83 \%$ females, 91\% white) at baseline and week 16 after treatment with an anti-IL6 receptor antibody in combination with MTX, as well as in serum of 77 donors (age; 42 (20-69), $51 \%$ females, $66 \%$ white). Marker
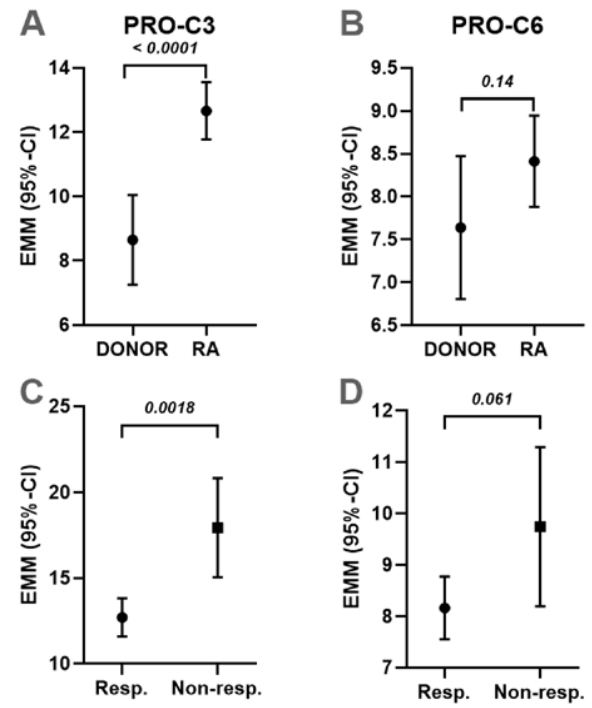

Fig. Difference in active fibrogenesis assessed by serum PRO-C3 and PRO-C6 between donors and RA patients at baseline $(A, B)$ and between resp. and non-resp. at week $16(C, D)$. Data is shown as back-transformed estimated marginal means (EMM) with 95\%-C adjusted for age, gender, and race (all), and BMI, disease duration and baseline marker level $(C, D)$. data was LN transformed. A general linear model was used when comparing groups.

Results: The serum fibrogenesis marker PRO-C3, but not PRO-C6, was significantly elevated in RA compared to donors $(2.1$ vs. $2.4 \approx 30 \%$ difference, $p<0.0001$, fig. $1 A$ ). None of the markers were correlated with disease measures such as DAS28, CRP, VASpain. None of the markers were modulated significantly in response to treatment. Interestingly, PRO-C3 levels were significantly higher at in non-responders (resp.) at week 16 compared to resp. (2.8 vs. $2.4 \approx$ $40 \%$ difference, $\mathrm{p}=0.0018$, fig. $1 \mathrm{C}$ ). Similar trend was observed for PRO-C6 (2.2 vs. $2.0 \approx 20 \%$ difference, $p=0.061$ fig. $1 D$ ).

Conclusion: Active fibrosis, with activated fibroblasts, may play an unseen role in RA. Patients will elevated levels of the fibrosis markers PRO-C3 and PRO-C6 were less likely to respond to an anti-IL6R. This may also give clue why such treatment are less efficacious in diseases with a clear fibrotic component.

REFERENCES:

[1] Karsdal et al. Rheumatoid arthritis: A case for personalized health care? ACR 2014; 66: 1273-80.

[2] Bay-Jensen et al. Effect of tocilizumab combined with methotrexate on circulating biomarkers of synovium, cartilage, and bone in the LITHE study. SAR 2014; 43: 470-8.

[3] Thudium et al. The Janus kinase $1 / 2$ inhibitor baricitinib reduces biomarkers of joint destruction in moderate to severe rheumatoid arthritis. ART 2020; 22.

[4] Drobinski et al. Connective tissue remodeling is differently modulated by tocilizumab versus methotrexate monotherapy in patients with early rheumatoid arthritis: the AMBITION study. ART 2021; 23.

[5] Karsdal et al. Profiling and targeting connective tissue remodeling in autoimmunity - A novel paradigm for diagnosing and treating chronic diseases. AutoRev 2021; 20.

Disclosure of Interests: None declared

DOI: 10.1136/annrheumdis-2021-eular.3619

\section{$\mathrm{AB} 0068$ \\ B- AND T- LYMPHOCYTE SUBSETS IN PATIENTS WITH EARLY AND PROGRESSED RHEUMATOID ARTHRITIS}

A. Martynova ${ }^{1}$, T. Popkova ${ }^{1}$, A. Aleksankin ${ }^{1}$, E. Gerasimova ${ }^{1}$. ${ }^{1}$ V.A. Nasonova Research Institute of Rheumatology, Systemic Rheumatic Diseases, Moscow, Russian Federation

Background: Role of B- and T- lymphocytes is well established in pathogenesis of rheumatoid arthritis (RA). Self-containing activation of B-cells in ectopic germinative centers is followed by auto-activation of T-lymphocytes while T-cells themselves are antigen-presenting cells for B-lymphocytes [1-2]. As these processes continue with the duration of RA, different subsets of $B$ - and T-cell might be prevalent at different stages of RA.

Objectives: Evaluate differences in dynamics of B- and T- cell subsets in early and progressed RA.

Methods: 53 patients with diagnosed RA(ACR/EULAR 2010 criteria) were included in research in October 2019 - May 2020. Age median 54,2 [47; 62] yrs. 2 groups were formed: 1 st ( $n=27$ pts., 25 female and 2 male) with early arthritis, median duration of RA $6[5 ; 12]$ months prior to therapy, mediane DAS28-CRP - 5,8 [5,15-6,2]; and 2nd group ( $\mathrm{n}=26$ pts., 22 female and 4 male) - patients with progressed RA, duration of RA 84 [24; 121] months, on DMARDs (methotrexate $20[15 ; 20] \mathrm{mg} /$ week or leflunomide $20 \mathrm{mg} /$ day), mediane DAS28-CRP - 6,31 [5,64-6,88]. Control group included 29 individuals (23 female and 6 male), age median $58,5[53 ; 62]$ yrs. Blood of all patients underwent standart flow cytometry with T- and B-cell immunotyping.

Results: B and C-cells subsets were studied. B-cells subsets are presented in table 1 below.

Conclusion: As disease activity was high in both groups, therefore therapy was ineffective, and results could be interpreted as natural progression of RA, resulting mainly in dynamics of switched B-memory cells, transitioning B-cells and plasmoblasts. These subsets might be indicating disease development patterns but further research is required.

\section{REFERENCES:}

[1] Weyand, C. M., \&Goronzy, J. J. Ectopic germinal center formation in rheumatoid synovitis. Annals of the New York Academy of Sciences, 2003, 987, 140-149.

[2] Shi K., HayashidaK. Lymphoid Chemokine B Cell-Attracting Chemokine-1 (CXCL13) Is Expressed in Germinal Center of Ectopic Lymphoid Follicles Within the Synovium of Chronic Arthritis Patients. J Immunol 2001; 166:650-655. 